\title{
A focal brain-cooling device as an alternative to electrical stimulation for language mapping during awake craniotomy: patient series
}

\author{
Sadahiro Nomura, MD, ${ }^{1}$ Takao Inoue, PhD, ${ }^{2}$ Hirochika Imoto, MD, ${ }^{1}$ Hirokazu Sadahiro, MD, ${ }^{1}$ Kazutaka Sugimoto, MD, ${ }^{1}$ \\ Yuichi Maruta, $\mathrm{PhD},{ }^{1}$ Hideyuki Ishihara, $\mathrm{MD},{ }^{1}$ and Michiyasu Suzuki, $\mathrm{MD}^{2}$
}

Departments of ${ }^{1}$ Neurosurgery and ${ }^{2}$ Advanced Thermal Neurobiology, Yamaguchi University School of Medicine, Ube, Yamaguchi, Japan

BACKGROUND Functional mapping in awake craniotomy has the potential risk of electrical stimulation-related seizure. The authors have developed a novel mapping technique using a brain-cooling device. The cooling probe is cylindrical in shape with a thermoelectric cooling plate $(10 \times 10 \mathrm{~mm})$ at the bottom. A proportional integration and differentiation-controlled system adjusts the temperature accurately (Japan patent no. P5688666). The authors used it in two patients with glioblastoma. Broca's area was identified by electrical stimulation, and then the cooling probe set at $5^{\circ} \mathrm{C}$ was attempted on it.

OBSERVATIONS Electrocorticogram was suppressed, and the temperature dropped to $8^{\circ} \mathrm{C}$ in $50 \mathrm{sec}$. A positive aphasic reaction was reproduced on Broca's area at a latency of $7 \mathrm{sec}$. A negative reaction appeared on the adjacent cortices despite the temperature decrease. The sensitivity and specificity were $60 \%$ and $100 \%$, respectively. No seizures or other adverse events related to the cooling were recognized, and no histological damage to the cooled cortex was observed.

LESSONS The cooling probe suppressed topographical brain function selectively and reversibly. Awake functional mapping based on thermal neuromodulation technology could substitute or compensate for the conventional electrical mapping.

https://thejns.org/doi/abs/10.3171/CASE21131

KEYWORDS brain temperature; awake surgery; translational research

Awake craniotomy for brain tumors near the functional cortex has been developed in clinical use since the $1990 \mathrm{~s}^{1,2}$ The guideline has almost been established, ${ }^{3}$ and the protocol has been refined. ${ }^{4,5}$ Language cortices are identified by language tasks while the area is being suppressed by electrical stimulation. ${ }^{6}$ One drawback to the procedure is that stimulation-related seizure occurs in the range of $0 \%-24 \%,{ }^{7,8}$ although a successful attempt for avoiding seizure including the train-of-five stimulation technique has been reported. ${ }^{9}$ This may cause several accidents, stop the identification of the functional area, and require changing of surgical strategy. Postoperative seizure by electrical stimulation for motor-evoked potential monitoring has been reported as well. ${ }^{10,11}$ In order to avoid complications, we have been developing a novel method of brain mapping by focal brain cooling as a substitute for mapping by electrical stimulation.

It is well known that hypothermia around $32-34^{\circ} \mathrm{C}$ helps protect the brain in patients experiencing postcardiac arrest ${ }^{12}$ or severe neurotrauma ${ }^{13-16}$ through the suppression of brain metabolism ${ }^{17}$ and glutamate release. ${ }^{18}$ We found that epileptic seizures terminated at $15^{\circ} \mathrm{C}$ or lower ${ }^{19-28}$ and found that neuronal function deteriorated at $10^{\circ} \mathrm{C}$ or lower. ${ }^{29,30}$ The threshold of low temperature for histological brain damage is not known exactly; however, irreversible damage occurred neither by continuous cooling at $5^{\circ} \mathrm{C}$ for 1 hour ${ }^{31}$ nor by cooling in repeats of $30 \mathrm{sec}$ at $5^{\circ} \mathrm{C}$ with a $1.5-\mathrm{min}$ interval for 2 hours. ${ }^{32}$ From these findings, it can be concluded that a temperature between $5^{\circ} \mathrm{C}$ and $10^{\circ} \mathrm{C}$ enables reversible suppression of brain function; therefore, it is optimal for functional mapping based on thermal neuromodulation technology.

In order to terminate a seizure, the cooling device should cover the epileptic focus with a margin. On the contrary, functional mapping requires accuracy in bordering. For this purpose, we decreased the side length of the square cooling plate of our device to $10 \mathrm{~mm}$, which is smaller than the gyrus width. The challenge we did not anticipate

ABBREVIATIONS GBM = glioblastoma multiforme; IQ = intelligence quotient; MRI = magnetic resonance imaging; PID = proportional integration and differentiation. INCLUDE WHEN CITING Published July 12, 2021; DOI: 10.3171/CASE21131.

SUBMITTED February 26, 2021. ACCEPTED May 14, 2021.

(C) 2021 The authors, CC BY-NC-ND 4.0 (http://creativecommons.org/licenses/by-nc-nd/4.0/). 


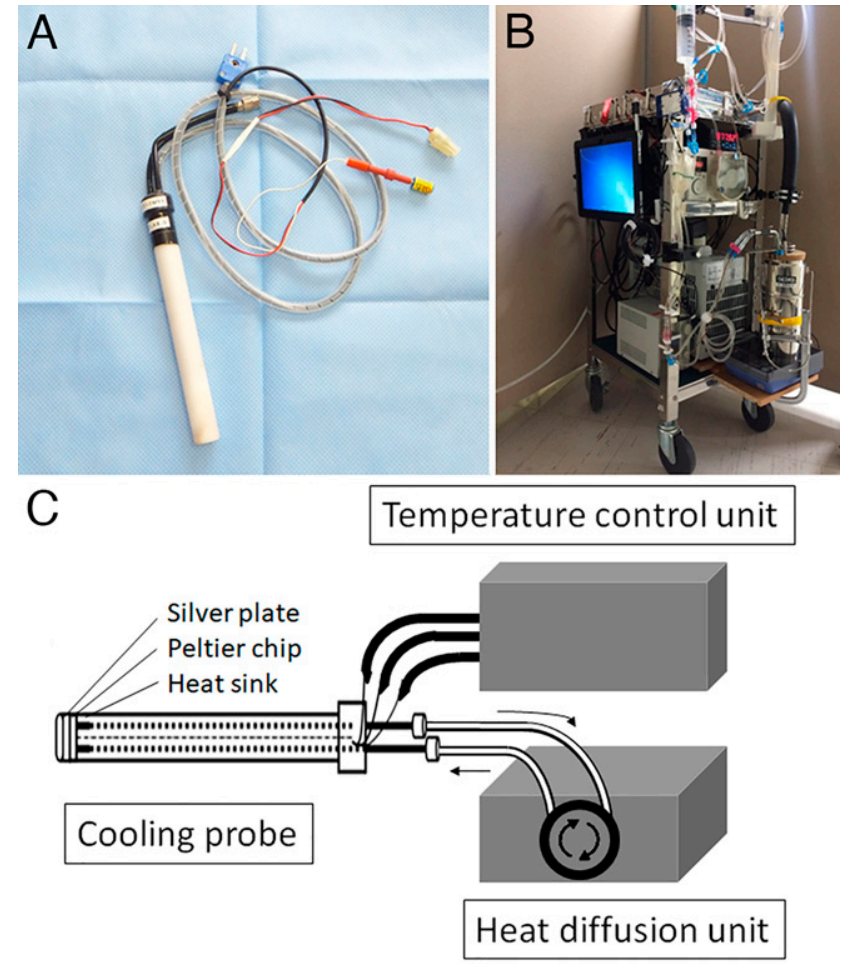

FIG. 1. Components of the cooling device. A cooling probe (A) has a thermoelectric Peltier chip with an attached silver plate on the cooling side and a silver heat sink on the other side. The temperature of the silver plate is controlled by a temperature control unit (B). C: The byproduct heat is collected to the heat sink and diffused by the perfused saline solution to and from a reservoir in the heat diffusion unit.

was that such a small area of cooling enabled the suppression of the topographical function superselectively and reproducibly. On the other hand, we were confident that a cooling device made with a Peltier chip should be one of the most optimal materials for rapid and accurate temperature control. We report two patients with intraoperative mapping of the language area using a focal brain-cooling device.

\section{Study Description \\ Patients}

Two patients with glioblastoma multiforme (GBM) in the left hemisphere were included in the study. The patients and their families agreed to participate in our study after being adequately informed of the aims, methods, and anticipated benefits, as well as the potential risks and discomfort associated with their involvement. All patients had the right to withdraw from the study at any given time.

\section{Cooling Device}

A proportional integration and differentiation (PID)-controlled cooling system for the focal brain was originally developed in our laboratory for the treatment of intractable epilepsy (Japan patent no. P5688666). The system consists of a handheld cooling probe (Fig. 1), temperature control unit, and heat diffusion unit. ${ }^{19,25,26}$ The probe is a cylindrical acrylic case (diameter, $16 \mathrm{~mm}$; length, $150 \mathrm{~mm}$ ) embedded in a thermoelectric Peltier chip $(10 \times 10 \mathrm{~mm}$; maximum current: $0.8 \mathrm{~A}$; maximum voltage: $8.8 \mathrm{~V}$; maximum cooling capacity: $4.2 \mathrm{~W}$; Ferrotec) with an attached silver plate $(10 \times 10 \mathrm{~mm})$ on the cylinder bottom and a heat sink inside the cylinder. A thermocouple is mounted on the silver plate so that the temperature control unit adjusts the temperature using PID technology. The byproduct heat is collected into the heat sink and is diffused by the perfused saline to and from a reservoir in the heat-diffusion unit.

The cooling probe was sterilized with ethylene oxide gas 2 days before surgery or hydrogen peroxide gas plasma 1 day before.

\section{Surgery and Mapping Procedure}

Craniotomy was performed under general anesthesia. The location of the tumor and central sulcus was identified using neuronavigation and somatosensory-evoked potentials. Patients were awakened, and the motor (Broca) and sensory (Wernicke) language areas were mapped using the language tasks under electrical stimulation. Then, the cooling probe set at $5^{\circ} \mathrm{C}$ was placed on the identified Broca's area and the cortex surrounding it in order to observe whether the patient's response was the same as that with the responses electrical mapping. Brain surface temperature was measured using a thermocouple affixed to the silver plate. The cortex, covering over the tumor and to be resected in the course of tumor resection, was cooled at $5^{\circ} \mathrm{C}$ for $5 \mathrm{~min}$ and treated as a histopathological specimen in order to observe the cooling influence in patient 2. Subcortical tract mapping was not performed. Tumor resection was performed based on the electrical brain mapping; therefore, the results of the surgery were not relevant to analyze the accuracy of the cooling mapping. In patient 2, the patient's voice of the number counting was recorded using a superdirective microphone AG-MC200 (Panasonic) and digitized with $48 \mathrm{kHz}$ of frequency and 16 bit of quantization rate.

The cooling system was used only in clinical research since it has not been submitted yet to the US Food and Drug Administration or the Japan Pharmaceuticals and Medical Devices Agency for review regarding clinical use.

\section{Illustrative Cases \\ Patient 1}

A 78-year-old man was admitted to our hospital because of a headache lasting for 2 months. Magnetic resonance imaging (MRI) revealed a ring-enhanced tumor of $44 \times 47 \times 31 \mathrm{~mm}$ in the left frontal subcortical area. He had no language problems but cognitive impairment with 68 in full intelligence quotient $(\mathrm{IQ}), 67$ in verbal $I Q$,

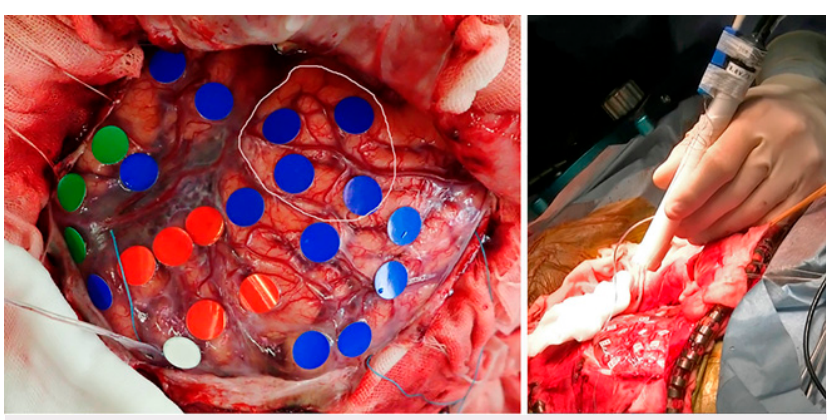

FIG. 2. Intraoperative photograph of patient 1. Left: Functional mapping by electrical stimulation. The red, green, white, and blue markings indicate the motor and sensory language areas, motor cortex, and areas with no response to the stimulation, respectively. The area in the circle indicates the tumor location. Right: The cooling probe is held by hand and approaches the brain surface. 


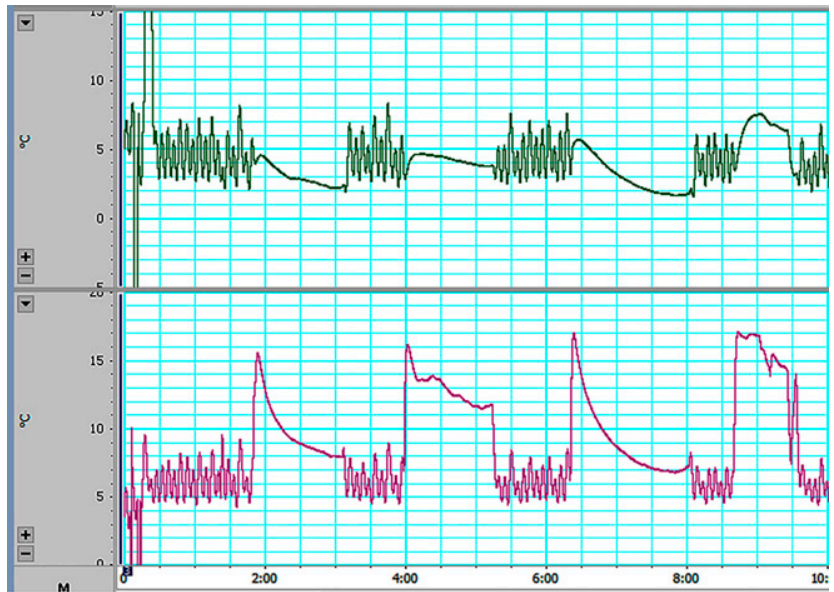

FIG. 3. Probe and brain temperatures in patient 1. The upper chart shows the temperature control of the probe. As the PID feedback control system attempts to maintain the probe temperature at $5^{\circ} \mathrm{C}$, the temperature fluctuates between $2^{\circ} \mathrm{C}$ and $8^{\circ} \mathrm{C}$. The lower chart indicates the brain temperature; however, the thermocouple measuring the temperature was placed on the probe surface, not on the brain. While the thermocouple was removed from the brain in the intervals between coolings, the temperatures were lower than during the cooling periods. The brain temperature reached $8^{\circ} \mathrm{C}$ in an average of 50 sec after the attachment of the probe. Among the four cooling trials, the first and third were successful; in the second trial, the probe had insufficient contact with the brain, and in the fourth trial, the delay in the temperature drop was due to the control system.

and 75 in performance IQ. Tumor removal was performed with awake craniotomy. After mapping with electrical stimulation (Fig. 2 left), the cooling probe set at $5^{\circ} \mathrm{C}$ (Fig. 3 upper) was placed on Broca's area and the surrounding cortices (Fig. 2 right). After an average of $50 \mathrm{sec}$ from the attachment of the probe, the brain temperature decreased to $8^{\circ} \mathrm{C}$ and then plateaued until the cooling end (Fig. 3 lower). A disturbance of picture naming occurred $7 \mathrm{sec}$ after the temperature reached $8^{\circ} \mathrm{C}$. The language function was disturbed three times during five successful coolings in the Broca's area, while no disturbance was noted with the cooling on the surrounding cortex. The sensitivity and specificity were $60 \%$ and $100 \%$, respectively. The Broca's area of the patient shifted posteriorly, so the tumor was totally resected without deficit of language function. No adverse effect of cooling was observed. Under the diagnosis of GBM, the patient received radiation therapy and chemotherapy and lived for 11 months since the onset of the symptom.

\section{Patient 2}

A 72-year-old man was emergently admitted because of epileptic seizures and aphasia. MRI revealed a ring-enhanced tumor of $35 \times$ $35 \times 40 \mathrm{~mm}$ in the left frontoparietotemporal area. Because the aphasia persisted, he was unable to name pictures before and during the awake craniotomy. After the mapping with electrical stimulation (Fig. 4 left), the cooling probe was placed on the Broca's area (Fig. 4 right) and the surrounding cortex. Only number counting was performed as the language task. Interruption of the number counting, the positive aphasic reaction in Broca's area, was observed three out of five times, and the negative reactions of the adjacent cortices were observed five out of five times. The sensitivity and specificity
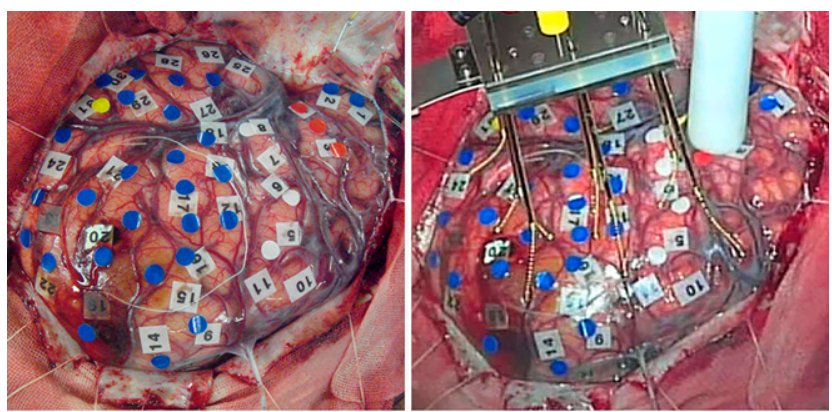

FIG. 4. Intraoperative photograph of patient 2. Left: Functional mapping by electrical stimulation. The red, yellow, white, and blue markings indicate the motor and sensory language areas, motor cortex, and areas with no response to the stimulation, respectively. The area in the circle indicates the tumor location. Right: The cooling probe was attached to the brain surface. Spring electrodes were prepared for observation after the discharge.

were $60 \%$ and $100 \%$, respectively. After functional mapping, the cortex on the tumor planned to be resected was cooled at $5^{\circ} \mathrm{C}$ for 5 min. Immediately after the cooling, the cortex was resected in 30 min, and the tumor was extracted. No histological damage in the cooled area was observed (Fig. 5). The tumor was subtotally resected. The patient mildly deteriorated in the aphasia postoperatively and recovered partly. No adverse events due to cooling were observed. Under the diagnosis of GBM, the patient received radiation therapy and chemotherapy and lived for 15 months since the onset of the symptoms.

\section{Discussion}

\section{Observations}

Awake functional mapping using the cooling technique was performed safely and successfully in both patients. The results of the histological tolerance seen in patient 2 proved that cooling at $5^{\circ} \mathrm{C}$ can also be applied to the brain area to be preserved. Using the same procedure with minimal modification of the temperature
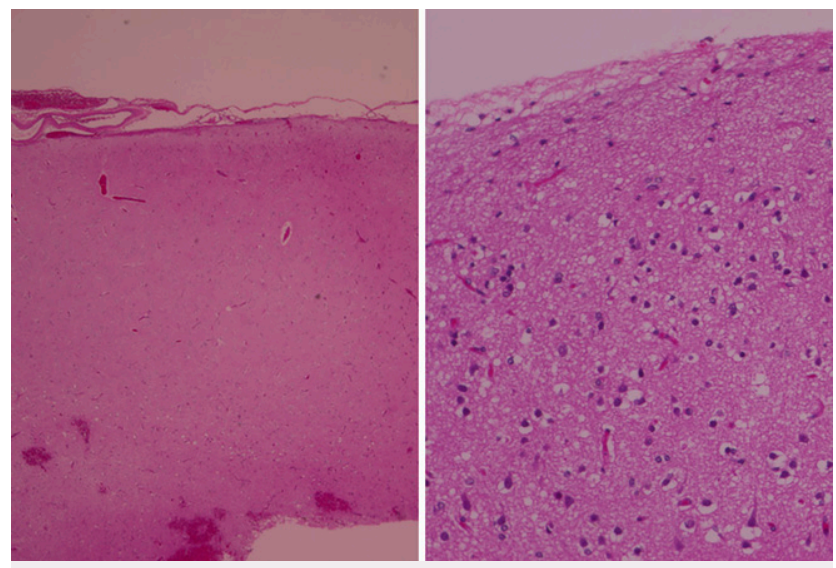

FIG. 5. Histology of the cooled cortex of the patient 2. Hematoxylin and eosin staining, low-power (left; original magnification $\times 40$ ) and high-power (right; original magnification $\times 200$ ) magnifications. No evident histological damage was observed. The cortex covering the glioblastoma was cooled at $5^{\circ} \mathrm{C}$ for $5 \mathrm{~min}$ before resection. 
control, we may be able to map the motor area, which is yet untried. Low-grade glioma will be more optimal for cooling mapping because of the difficulty in the determination of the resection bor$\operatorname{der}^{5,33}$ as compared with high-grade glioma.

Unlike electrical stimulation, cooling has no potential risk of intraoperative epileptic seizures. In the present study, we found no necessity for the spring electrodes that monitor after-discharge; thus, no space interference of the mapping probe and the electrodes occurred in the surgical field. This safe cooling-mapping method could substitute or compensate for electrical stimulation mapping, especially in the mapping of higher brain function, which requires a longer period of task and suppression. ${ }^{34} \mathrm{Com}$ pared with the point-touch of the electrical stimulation probe, the cooling probe touches the brain in a two-dimensional plane. It is supposed to be inaccurate in the determination of the border; however, the cooling probe suppresses a narrower area as compared with the electrical probe. The reason is that the cooling effect does not spread like electrical potentials, and the temperature gradient in the regions surrounding the probe was steep. ${ }^{29,30}$ Therefore, a false-positive response was unlikely to occur, as shown in the two patients.

Sensitivities in the exploration of the Broca's area were below $100 \%$. Single or any positive reactions in multiple attempts should be determined positive based on the results of the present study. The interval from the start of cooling until the appearance of the aphasia needed $1 \mathrm{~min}$. This is the latency in which the cooling device at $5^{\circ} \mathrm{C}$ decreases the brain temperature to $8^{\circ} \mathrm{C}$, since the brain is kept warmer by the circulation of blood. The latency was longer than when electrical stimulation was used. These drawbacks are the reasons why the reliability and usefulness of cooling are so far inferior to electrical mapping. Further investigation of the cooling technique and gaining cooling power could increase the level of sensitivity and shorten the reaction period to that of electrical mapping.

\section{Lessons}

We developed a novel brain mapping technique using a thermoelectric chip mounted probe. Within $50 \mathrm{sec}$ from the start of cooling, the temperature of Broca's cortex dropped to $8^{\circ} \mathrm{C}$ and motor aphasia appeared in $7 \mathrm{sec}$. No aphasia appeared when the adjacent cortex was cooled. No seizure occurred, and no histopathological damage on the cooled cortex was observed. The selective, reversible, and safe suppression of brain function by focal brain cooling possibly compensates for electrical mapping in awake craniotomy.

\section{Acknowledgments}

We thank Dr. Masami Fujii, Dr. Hiroshi Fujioka, Dr. Takashi Saito, Dr. Koji Kajiwara, and Dr. Koichi Yoshikawa, who invented the localized cerebrum cooling probe and brain function-mapping device. This work was supported by Grants-in-Aid for Scientific Research $S$ from the Japan Society for the Promotion of Science (grant no. $15 \mathrm{H} 05719$ to M.S.); a Grant for Medical Research and Development Programs Focused on Technology Transfers from the Japan Agency for Medical Research and Development (M.S.); a Grant for Regional Innovation Ecosystems from Ministry of Education, Culture, Sports, Science and Technology (M.S.); Grants-in-Aid for Scientific Research C from the Japan Society for the Promotion of Science (grant no. $19 \mathrm{~K} 06956$ to S.N.); and Grants-in-Aid for Scientific Research C from the Japan Society for the Promotion of Science (grant no. $20 \mathrm{~K} 09349$ to T.I.).

\section{References}

1. Berger MS, Ojemann GA, Lettich E. Neurophysiological monitoring during astrocytoma surgery. Neurosurg Clin N Am. 1990;1(1):65-80.

2. Ojemann G, Ojemann J, Lettich E, Berger M. Cortical language localization in left, dominant hemisphere. An electrical stimulation mapping investigation in 117 patients. J Neurosurg. 1989;71(3): 316-326.

3. Kayama T. The guidelines for awake craniotomy guidelines committee of the Japan awake surgery conference. Neurol Med Chir (Tokyo). 2012;52(3):119-141.

4. Duffau $H$, Capelle L, Denvil D, et al. Usefulness of intraoperative electrical subcortical mapping during surgery for low-grade gliomas located within eloquent brain regions: functional results in a consecutive series of 103 patients. J Neurosurg. 2003;98(4):764-778.

5. Soffietti R, Baumert BG, Bello L, et al. Guidelines on management of low-grade gliomas: report of an EFNS-EANO Task Force. Eur $J$ Neurol. 2010;17(9):1124-1133.

6. Szelenyi A, Bello L, Duffau H, et al. Intraoperative electrical stimulation in awake craniotomy: methodological aspects of current practice. Neurosurg Focus. 2010;28(2):E7

7. Eseonu Cl, Rincon-Torroella J, Lee YM, et al. Intraoperative seizures in awake craniotomy for perirolandic glioma resections that undergo cortical mapping. J Neurol Surg A Cent Eur Neurosurg. 2018;79(3):239-246.

8. Roca E, Pallud J, Guerrini F, et al. Stimulation-related intraoperative seizures during awake surgery: a review of available evidences. Neurosurg Rev. 2020;43(1):87-93

9. Szelényi A, Joksimovic $B$, Seifert V. Intraoperative risk of seizures associated with transient direct cortical stimulation in patients with symptomatic epilepsy. J Clin Neurophysiol. 2007;24(1):39-43.

10. Davis SF, Altstadt T, Flores R, et al. Report of seizure following intraoperative monitoring of transcranial motor evoked potentials. Ochsner J. 2013;13(4):558-560.

11. Ulkatan S, Jaramillo AM, Téllez MJ, et al. Incidence of intraoperative seizures during motor evoked potential monitoring in a large cohort of patients undergoing different surgical procedures. $J$ Neurosurg. 2017;126(4):1296-1302.

12. Hypothermia after Cardiac Arrest Study Group. Mild therapeutic hypothermia to improve the neurologic outcome after cardiac arrest. N Engl J Med. 2002;346(8):549-556.

13. Marion DW, Penrod LE, Kelsey SF, et al. Treatment of traumatic brain injury with moderate hypothermia. $N$ Engl J Med. 1997;336(8):540-546.

14. Suehiro E, Fujisawa $H$, Koizumi $H$, et al. Significance of differences between brain temperature and core temperature $(\delta T)$ during mild hypothermia in patients with diffuse axonal injury. Neurol Med Chir (Tokyo). 2011:51(8):551-555.

15. Suehiro E, Koizumi $H$, Fujisawa $H$, et al. Diverse effects of hypothermia therapy in patients with severe traumatic brain injury based on the computed tomography classification of the traumatic coma data bank. J Neurotrauma. 2015;32(5):353-358.

16. Suehiro E, Koizumi H, Fujiyama Y, Suzuki M. Recent advances and future directions of hypothermia therapy for traumatic brain injury. Neurol Med Chir (Tokyo). 2014;54(11):863-869.

17. Erecinska M, Thoresen M, Silver IA. Effects of hypothermia on energy metabolism in mammalian central nervous system. J Cereb Blood Flow Metab. 2003;23(5):513-530.

18. Shima $H$, Fujisawa $H$, Suehiro $E$, et al. Mild hypothermia inhibits exogenous glutamate-induced increases in nitric oxide synthesis. J Neurotrauma. 2003;20(11):1179-1187.

19. Fujii M, Fujioka H, Oku T, et al. Application of focal cerebral cooling for the treatment of intractable epilepsy. Neurol Med Chir (Tokyo). 2010;50(9):839-844.

20. Hata K, Fujiwara K, Inoue T, et al. Epileptic seizure suppression by focal brain cooling with recirculating coolant cooling system: 
modeling and simulation. IEEE Trans Neural Syst Rehabil Eng. 2019;27(2):162-171.

21. Hata K, Fujiwara K, Kano M, et al. Design of focal brain cooling system for suppressing epileptic seizures. Annu Int Conf IEEE Eng Med Biol Soc. 2017;2017:283-286.

22. Imoto H, Fujii M, Uchiyama J, et al. Use of a Peltier chip with a newly devised local brain-cooling system for neocortical seizures in the rat. Technical note. J Neurosurg. 2006;104(1):150-156.

23. Inoue T, Fujii M, Kida H, et al. Epidural focal brain cooling abolishes neocortical seizures in cats and non-human primates. Neurosci Res. 2017;122:35-44.

24. Kida H, Fujii M, Inoue T, et al. Focal brain cooling terminates the faster frequency components of epileptic discharges induced by penicillin G in anesthetized rats. Clin Neurophysiol. 2012;123(9): 1708-1713.

25. Nomura S, Fujii M, Inoue T, et al. Changes in glutamate concentration, glucose metabolism, and cerebral blood flow during focal brain cooling of the epileptogenic cortex in humans. Epilepsia. 2014; 55(5):770-776.

26. Nomura $\mathrm{S}$, Inoue $\mathrm{T}$, Imoto $\mathrm{H}$, et al. Effects of focal brain cooling on extracellular concentrations of neurotransmitters in patients with epilepsy. Epilepsia. 2017;58(4):627-634.

27. Nomura S, Kida H, Hirayama Y, et al. Reduction of spike generation frequency by cooling in brain slices from rats and from patients with epilepsy. J Cereb Blood Flow Metab. 2019;39(11):2286-2294.

28. Tanaka N, Fujii M, Imoto H, et al. Effective suppression of hippocampal seizures in rats by direct hippocampal cooling with a Peltier chip. J Neurosurg. 2008;108(4):791-797.

29. Fujii M, Inoue T, Nomura S, et al. Cooling of the epileptic focus suppresses seizures with minimal influence on neurologic functions. Epilepsia. 2012;53(3):485-493.

30. He Y, Inoue T, Nomura S, et al. Limitations of local brain cooling on generalized motor seizures from unknown foci in awake rats. Neurol Med Chir (Tokyo). 2019;59(4):147-153.

31. Oku T, Fujii M, Tanaka N, et al. The influence of focal brain cooling on neurophysiopathology: validation for clinical application. $J$ Neurosurg. 2009;110(6):1209-1217.

32. Yang XF, Kennedy BR, Lomber SG, et al. Cooling produces minimal neuropathology in neocortex and hippocampus. Neurobiol Dis. 2006;23(3):637-643.

33. Berger MS, Deliganis AV, Dobbins J, Keles GE. The effect of extent of resection on recurrence in patients with low grade cerebral hemisphere gliomas. Cancer. 1994;74(6):1784-1791.

34. Motomura K, Chalise L, Ohka F, et al. Supratotal resection of diffuse frontal lower grade gliomas with awake brain mapping, preserving motor, language, and neurocognitive functions. World Neurosurg. 2018;119:30-39.

\section{Disclosures}

The authors report no conflict of interest concerning the materials or methods used in this study or the findings specified in this paper.

\section{Author Contributions}

Conception and design: Nomura, Suzuki. Acquisition of data: Nomura, Inoue, Sadahiro, Sugimoto, Maruta, Suzuki. Analysis and interpretation of data: Nomura, Maruta, Ishihara, Suzuki. Drafting the article: Nomura. Critically revising the article: Nomura, Sugimoto. Reviewed submitted version of manuscript: Nomura, Inoue, Imoto, Sugimoto, Ishihara. Approved the final version of the manuscript on behalf of all authors: Nomura. Statistical analysis: Nomura. Administrative/technical/material support: Nomura. Study supervision: Nomura.

\section{Supplemental Information}

Previous Presentations

This manuscript is original, and the work was presented as a poster at the 78th Annual Meeting of Japan Neurosurgical Society, Osaka, Japan, October 9-12, 2019.

\section{Patient Informed Consent}

The necessary patient informed consent was obtained in this study.

\section{Correspondence}

Sadahiro Nomura: Yamaguchi University School of Medicine, Ube, Yamaguchi, Japan. snomura@yamaguchi-u.ac.jp. 